\title{
Modes of nonlinear acoustic transparency in the strained paramagnetic crystal
}

\author{
S.V. Sazonov* \\ I. Kant State University of Russia, 236041 Kaliningrad, Russia \\ N.V. Ustinor \\ Tomsk State University, 634050 Tomsk, Russia
}

(Dated: July 16, 2018)

\begin{abstract}
The propagation of the transverse-longitudinal acoustic pulses through a strained cubic crystal containing the resonant paramagnetic impurities with effective spin $S=1$ is investigated. It is supposed that the pulses propagate under arbitrary angle with respect to the direction of the external static deformation parallel to the fourth-order symmetry axis. In this geometry, both the transverse and longitudinal components of the acoustic field have the high-frequency and zero-frequency spectral components. We show that a pulse can propagate in the modes different from the acoustic self-induced transparency. In particular, a pulse propagating in the mode of acoustic self-induced supertransparency substantially changes the populations of the spin sublevels, but its group velocity remains almost equal to the linear velocity of the sound. If a pulse propagates in the acoustic extraordinary transparency mode, then its group velocity is substantially lower while the sublevel populations remain virtually invariant. Also, the modes of propagation under conditions of weakly excited spin transitions and large detuning of the pulse high-frequency components are identified.
\end{abstract}

PACS numbers: $43.25 .+\mathrm{y}, 43.35 .+\mathrm{d}$

\section{INTRODUCTION}

Historically, the coherent optical effects found their acoustic analogues after a while. Such a correspondence is easily traced, for instance, in the case of the selfinduced transparency (SIT) phenomenon. Its discovery in 1967 [1] and theoretical explanation in 1969 [2] stimulated a search for the acoustic analogue. The experimental observation at the liquid helium temperature of the acoustic self-induced transparency (ASIT) of the longitudinal hypersound in cubic crystal $\mathrm{MgO}$ containing paramagnetic ions $\mathrm{Fe}^{2+}$ and $\mathrm{Ni}^{2+}$ was carried out in 1970 by Shiren [3], who gave also the theoretical consideration of this effect. Independently, ASIT was studied theoretically for transverse hypersound in a system of paramagnetic impurities with effective spin $S=1 / 2$ [4]. Soon after that, this effect was successfully revealed in a crystal $\mathrm{LiNbO}_{3}$ alloyed by ions $\mathrm{Fe}^{2+}[5]$.

Despite the existence of the optical-acoustic analogies mentioned above, the acoustic coherent effects do not appear as the exact copies of optical ones. Obviously, the quantitative differences connected with a great distinction of the light and sound velocities or the frequencies of the electric-dipole and spin-phonon transitions take place. It should be stressed that the qualitative differences exist there. Thus, the optical wave is especially transverse whereas the acoustic one has the longitudinaltransverse structure.

The interaction between transverse and longitudinal components of the acoustic pulse in a crystal without

\footnotetext{
*Electronic address: barab@newmail.ru
}

†Electronic address: nustinov@mail.ru anharmonicity of the lattice modes happens due to a presence of paramagnetic impurities. Its influence on the dynamics of the components is inessential, if the linear velocities of acoustic waves differ significantly, as it takes place in the most solids. However, the linear velocities of transverse and longitudinal sounds are close in the ion crystals of halogenide of alkaline metals [6]. Since the components of the strain field interact effectively in this case, new features of their dynamics have to be expected. Indeed, new mode of the resonant acoustic transparency has been revealed theoretically in Ref. 7. The propagation of the transverse-longitudinal elastic pulses in this mode is accompanied by trapping of the population of the spin sublevels. Nevertheless, the deceleration in the velocity of the pulses is comparable to that in the case of ASIT of especially longitudinal or transverse hypersound [3, 4].

The theoretical considerations of the nonlinear dynamics of transverse-longitudinal acoustic pulses were fulfilled basically for the paramagnetic ions with effective spin $S=1 / 2$ 7, 8, 9]. At the same time, it is well-known that the strongest interaction with the crystal lattice oscillations takes place in the case $S=1$ [6]. Since the spin-phonon interaction is greater here on two or three orders than for $S=1 / 2$, the investigation of acoustic transparency of transverse-longitudinal pulses in a system of paramagnetic impurities with effective spin $S=1$ is more preferable from the point of view of experimental testing.

The resonant modes of propagation of the transverselongitudinal hypersound in direction parallel to external magnetic field and an axis of symmetry of the fourth order of the paramagnetic crystal with $S=1$ have been studied in 10]. It was shown that these modes coincide with ones discussed in Ref. 7. The distinctive feature of 
the geometry considered there is that the roles of transverse and longitudinal components of the acoustic pulses are strictly various: the former component excites the spin-phonon transitions in the Zeeman triplet, the latter one causes the dynamic shifts of the frequencies of these transitions.

Recently, it has been shown in the case of especially longitudinal acoustic pulses propagating under arbitrary angle with respect to an axis of the symmetry of the paramagnetic crystal, that both functions can be carried out by a single component of the strain field [11]. In this connection, the studying of the nonlinear modes of the acoustic transparency for the transverse-longitudinal pulses that propagate in a system of the effective spins $S=1$ under arbitrary direction to the symmetry axis is of great interest. The present report is devoted to allocating these modes. Following to Ref. 11, we suppose here that the external magnetic field is absent, and splitting of the energy sublevels is created by the static deformation of the paramagnetic crystal. For this reason, the degeneration of the spin states is removed incompletely in contrast to usual ASIT.

The paper is organized as follows. In Section II, the system of material and wave equations describing the interaction in the strained crystal of the transverselongitudinal elastic pulses with the paramagnetic impurities having effective spin $S=1$ is derived. We use the semi-classical approach, i.e., the dynamics of the acoustic fields is assumed to obey the classical Hamilton equations for the continuous medium, but the spin subsystem is treated as a quantum object. The order of the derivatives in the wave equations on the strain field components is reduced with the help of the unidirectional propagation (UP) approximation. In Section III, the approximation of slowly varying envelopes (SVE) is applied to the particular case of the system obtained in the previous section. It is shown that the evolution of the acoustic pulses in the paramagnetic crystal is governed by the well-studied equations of the long/shortwave coupling (LSWC) 12, 13. The pulse solutions decreasing exponentially and rationally of the LSWC equations are presented in Section IV. These solutions are exploited to classify the modes of the acoustic transparency of transverse-longitudinal pulses in the deformed paramagnetic crystal.

\section{BASIC MODEL}

Consider a cubic crystal containing the paramagnetic impurities with effective spin $S=1$. Let a transverselongitudinal acoustic pulse propagate in the crystal along the $z$ axis directed under arbitrary angle $\alpha$ with respect to one of its axes of symmetry of the fourth-order $\left(z^{\prime}\right.$ axis; see Fig. 1). Also, we assume that an external field of static and uniform deformation employing the crystal is parallel to the $z^{\prime}$ axis.

The Hamiltonian $\hat{H}$ of the spin-elastic interaction that

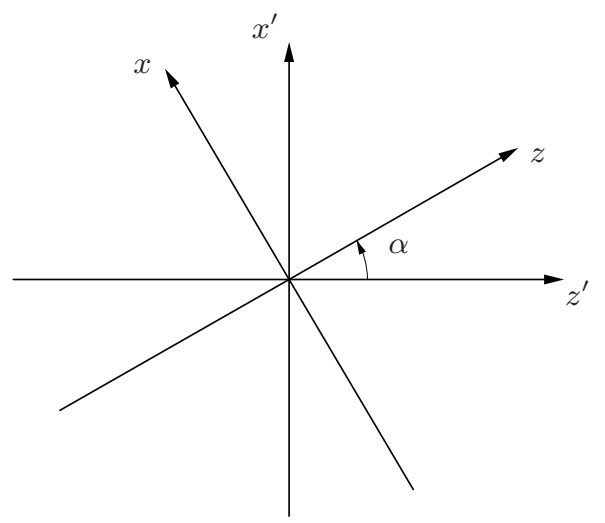

FIG. 1: Scheme of the coordinate axes.

arises in the most general case 14 is a function of the bilinear combination of spin operators $\hat{S}_{j}\left(j=x^{\prime}, y, z^{\prime}\right)$ :

$$
\hat{H}=f\left(\hat{S}_{i} Q_{i j} \hat{S}_{j}\right) .
$$

Coefficients $Q_{i j}$ in this formula are supposed to depend on the components of the strain tensor

$$
\mathcal{E}_{m l}=\frac{1}{2}\left(\frac{\partial U_{m}}{\partial x_{l}}+\frac{\partial U_{l}}{\partial x_{m}}\right)
$$

$\left(m, l=x^{\prime}, y, z^{\prime} ; U_{m}\right.$ are the components of the displacement vector $\mathbf{U})$. In a representation of the eigenfunctions of operator $\hat{S}_{z^{\prime}}$, the spin matrices take the form [6]

$$
\begin{gathered}
\hat{S}_{x^{\prime}}=\frac{1}{\sqrt{2}}\left(\begin{array}{lll}
0 & 1 & 0 \\
1 & 0 & 1 \\
0 & 1 & 0
\end{array}\right), \quad \hat{S}_{y}=\frac{i}{\sqrt{2}}\left(\begin{array}{ccc}
0 & -1 & 0 \\
1 & 0 & -1 \\
0 & 1 & 0
\end{array}\right), \\
\hat{S}_{z^{\prime}}=\left(\begin{array}{ccc}
1 & 0 & 0 \\
0 & 0 & 0 \\
0 & 0 & -1
\end{array}\right) .
\end{gathered}
$$

The coefficients of the bilinear combination satisfy condition $Q_{i j}=\delta_{i j}\left(\delta_{i j}\right.$ is the Kronecker symbol) if the external deformation of the crystal is absent. Expanding these coefficients and function $f$ in the power series on $\mathcal{E}_{m l}$ and retaining the first order terms, we obtain

$$
f=f\left(\hat{S}^{2}\right)+f^{\prime}\left(\hat{S}^{2}\right)\left(\frac{\partial Q_{i j}}{\partial \mathcal{E}_{m l}}\right)_{0} \hat{S}_{i} \hat{S}_{j} \mathcal{E}_{m l},
$$

where subscript "0" means that the values of derivatives correspond to nondeformed crystal. The first term depending on the Kazimir operator $\hat{S}^{2}=S(S+1)=2$ is omitted in what follows as the constant addition to $\hat{H}$. Then, Hamiltonian (10) is expressed through the components of the spin-elastic interaction tensor

$$
G_{i j l m}=f^{\prime}\left(\hat{S}^{2}\right)\left(\frac{\partial Q_{i j}}{\partial \mathcal{E}_{m l}}\right)_{0}
$$

as given

$$
\hat{H}=\frac{1}{2} G_{i j l m} \mathcal{E}_{m l}\left(\hat{S}_{i} \hat{S}_{j}+\hat{S}_{j} \hat{S}_{i}\right)
$$


In the case of the geometry we consider, the last equation is rewritten in the following manner

$$
\hat{H}=\hat{H}_{0}+\hat{V},
$$

where $\hat{H}_{0}$ is the Hamiltonian of the effective spin in the field $\mathcal{E}_{z^{\prime} z^{\prime}}^{(0)}$ of the external deformation:

$$
\hat{H}_{0}=G_{\|} \mathcal{E}_{z^{\prime} z^{\prime}}^{(0)} \hat{S}_{z^{\prime}}^{2}=\hbar \omega_{0}\left(\begin{array}{lll}
1 & 0 & 0 \\
0 & 0 & 0 \\
0 & 0 & 1
\end{array}\right),
$$

$\hat{V}$ is the Hamiltonian of interaction between the effective spin and strain field of the acoustic pulse propagating through the crystal [14]:

$$
\begin{array}{r}
\hat{V}=G_{\|} \mathcal{E}_{z z} \hat{S}_{z}^{2}+\frac{G_{\perp}}{2}\left[\mathcal{E}_{x z}\left(\hat{S}_{x} \hat{S}_{z}+\hat{S}_{z} \hat{S}_{x}\right)\right. \\
\left.+\mathcal{E}_{y z}\left(\hat{S}_{y} \hat{S}_{z}+\hat{S}_{z} \hat{S}_{y}\right)\right] .
\end{array}
$$

Here we use notations $G_{\|}=G_{z^{\prime} z^{\prime} z^{\prime} z^{\prime}}=G_{x^{\prime} x^{\prime} x^{\prime} x^{\prime}}=$ $G_{y y y y}, \omega_{0}=G_{||} \mathcal{E}_{z^{\prime} z^{\prime}}^{(0)} / \hbar$ ( $\hbar$ is the Planck constant) and $G_{\perp}=G_{x^{\prime} x^{\prime} z^{\prime} z^{\prime}}=G_{x^{\prime} x^{\prime} y y}=G_{y y z^{\prime} z^{\prime}}$. Operators $\hat{S}_{x}$ and $\hat{S}_{z}$ are connected with $\hat{S}_{x^{\prime}}$ and $\hat{S}_{z^{\prime}}$ by means of the transformation of rotation $[3,15$

$$
\begin{aligned}
& \hat{S}_{x}=\hat{S}_{x^{\prime}} \cos \alpha-\hat{S}_{z^{\prime}} \sin \alpha, \\
& \hat{S}_{z}=\hat{S}_{z^{\prime}} \cos \alpha+\hat{S}_{x^{\prime}} \sin \alpha .
\end{aligned}
$$

For the consideration of the dynamics of the effective spins and the acoustic pulse to be self-consistent, we introduce the Hamiltonian of the elastic field

$$
\begin{aligned}
H_{a}= & \frac{1}{2} \int\left\{\frac{p_{x}^{2}+p_{y}^{2}+p_{z}^{2}}{\rho}+\rho a_{\|}^{2}\left(\frac{\partial U_{z}}{\partial z}\right)^{2}\right. \\
& \left.+\rho a_{\perp}^{2}\left[\left(\frac{\partial U_{x}}{\partial z}\right)^{2}+\left(\frac{\partial U_{y}}{\partial z}\right)^{2}\right]\right\} d \mathbf{r},
\end{aligned}
$$

where $\rho$ is the mean crystal density; $p_{x}, p_{y}$ and $p_{z}$ are the components of momentum density of the local displacement of the crystal; $a_{\|}$and $a_{\perp}$ are linear velocities of the longitudinal and transversal hypersounds, respectively. We assume in (6) that all dynamical displacements $U_{x}$, $U_{y}$ and $U_{z}$ depend on variables $z$ and $t$ only. The integral is taken over the crystal volume.

In accordance with the general scheme of the semiclassical approach 14, 16], we describe evolution of the effective spin by the equation on the density matrix $\hat{\rho}$ :

$$
i \hbar \frac{\partial \hat{\rho}}{\partial t}=\left[\hat{H}_{0}+\hat{V}, \hat{\rho}\right] .
$$

At the same time, dynamics of the acoustic field obey the classical Hamilton equations for the continuous medium:

$$
\begin{gathered}
\frac{\partial \mathbf{p}}{\partial t}=-\frac{\delta}{\delta \mathbf{U}}\left(H_{a}+<\hat{\tilde{V}}>\right), \\
\frac{\partial \mathbf{U}}{\partial t}=\frac{\delta}{\delta \mathbf{p}}\left(H_{a}+<\hat{\tilde{V}}>\right),
\end{gathered}
$$

where

$$
<\hat{\tilde{V}}>=\int n<\hat{V}>d \mathbf{r},
$$

$n$ is the concentration of the paramagnetic impurities; $<\hat{V}>=\operatorname{Tr}(\hat{\rho} \hat{V})$ is the quantum average of $\hat{V}$.

Let the density matrix be represented in the next form

$$
\hat{\rho}=\left(\begin{array}{lll}
\rho_{33} & \rho_{32} & \rho_{31} \\
\rho_{23} & \rho_{22} & \rho_{21} \\
\rho_{13} & \rho_{12} & \rho_{11}
\end{array}\right) .
$$

Eqs. (2), (4) and (5) give us the following expression for the Hamiltonian of the spin-phonon interaction:

$$
\hat{V}=\left(\begin{array}{ccc}
V_{11} & -V_{12}^{*} & V_{13}^{*} \\
-V_{12} & V_{22} & V_{12}^{*} \\
V_{13} & V_{12} & V_{11}
\end{array}\right),
$$

where

$$
\begin{gathered}
V_{11}=\frac{G_{\|}}{2}\left(1+\cos ^{2} \alpha\right) \mathcal{E}_{z z}-\frac{G_{\perp}}{2} \sin 2 \alpha \mathcal{E}_{x z}, \\
V_{22}=G_{\| \mid} \sin ^{2} \alpha \mathcal{E}_{z z}+G_{\perp} \sin 2 \alpha \mathcal{E}_{x z}, \\
V_{12}=-\frac{G_{\| \mid} \sin 2 \alpha}{2 \sqrt{2}} \mathcal{E}_{z z}-\frac{G_{\perp}}{\sqrt{2}}\left(\cos 2 \alpha \mathcal{E}_{x z}+i \cos \alpha \mathcal{E}_{y z}\right), \\
V_{13}=\frac{G_{\|}}{2} \sin ^{2} \alpha \mathcal{E}_{z z}+G_{\perp}\left(\sin 2 \alpha \mathcal{E}_{x z} / 2+i \sin \alpha \mathcal{E}_{y z}\right) .
\end{gathered}
$$

The physical mechanism of spin-elastic interaction in the case considered is the Van Vleck mechanism [6]. The strain fields modulate the intracrystalline electric field in a location of the paramagnetic ions. Due to the quadrupole Stark effect, the static gradient of the electric field causes a splitting of the quantum sublevels of effective spin $S=1$ that occur to be degenerate on the absolute value of its projection $S_{z^{\prime}}$ (see (3) and Fig. 2). The components of the acoustic pulse excite the electroquadrupole transitions between these sublevels and, as it follows from the expressions for $V_{11}$ and $V_{22}$, shift dynamically the transition frequency.

Using (6) and (8)- (10), we deduce the wave equations on the strain field components:

$$
\begin{array}{r}
\frac{\partial^{2} \mathcal{E}_{z z}}{\partial t^{2}}-a_{\|}^{2} \frac{\partial^{2} \mathcal{E}_{z z}}{\partial z^{2}}=\frac{n G_{\|}}{2 \rho} \frac{\partial^{2}}{\partial z^{2}}\left[\left(3 \sin ^{2} \alpha-2\right) \rho_{22}\right. \\
+\frac{\sin 2 \alpha}{\sqrt{2}}\left(\rho_{23}+\rho_{32}-\rho_{12}-\rho_{21}\right) \\
\left.+\sin ^{2} \alpha\left(\rho_{13}+\rho_{31}\right)\right] \\
\frac{\partial^{2} \mathcal{E}_{x z}}{\partial t^{2}}-a_{\perp}^{2} \frac{\partial^{2} \mathcal{E}_{x z}}{\partial z^{2}}=\frac{n G_{\perp}}{4 \rho} \frac{\partial^{2}}{\partial z^{2}}\left[3 \sin 2 \alpha \rho_{22}\right. \\
+\sqrt{2} \cos 2 \alpha\left(\rho_{23}+\rho_{32}-\rho_{12}-\rho_{21}\right) \\
\left.+\sin 2 \alpha\left(\rho_{13}+\rho_{31}\right)\right]
\end{array}
$$




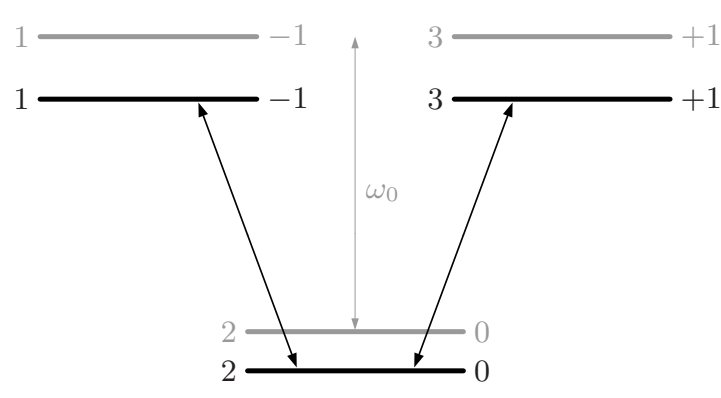

FIG. 2: Splitting of spin sublevels in the field of the static deformation (gray color), their position in a presence of the acoustic pulse and quantum transitions excited. The number of the level and the corresponding value of projection $S_{z^{\prime}}$ of effective spin on the axis of the external deformation are indicated from the left and right, respectively.

$$
\begin{aligned}
\frac{\partial^{2} \mathcal{E}_{y z}}{\partial t^{2}}-a_{\perp}^{2} \frac{\partial^{2} \mathcal{E}_{y z}}{\partial z^{2}} & =i \frac{n G_{\perp}}{2 \rho} \frac{\partial^{2}}{\partial z^{2}}\left[\sin \alpha\left(\rho_{31}-\rho_{13}\right)\right. \\
+ & \left.\frac{\cos \alpha}{\sqrt{2}}\left(\rho_{12}+\rho_{32}-\rho_{21}-\rho_{23}\right)\right] .
\end{aligned}
$$

Equations (77) and (11)-(13) form the self-consistent system describing the nonlinear dynamics of transverselongitudinal acoustic pulses in the deformed crystal containing the paramagnetic impurities with effective spin $S=1$. Since its analysis is very complicated in the general case, we restrict subsequent consideration by a specific model.

Let us assume that the linear velocities of both acoustic waves are equal:

$$
a_{\|}=a_{\perp}=a .
$$

This restriction is rather artificial, since $a_{\|}>a_{\perp}$ in solids. As noted in the previous section, it is fulfilled best of all in the ion crystals of halogenide of alkaline metals 6]. One of the representatives of such the crystals is, for instance, $\mathrm{NaBr}$ [17]. Using typical experimental parameters of medium and acoustic pulses, we show at the end of Section IV that strict observance of this condition is not very important from physical point of view. The case of the acoustic pulse propagation through deformed paramagnetic crystal with effective spin $S=1$, in which the linear velocities of the components differ essentially, has been studied in Ref. 11 .

As far as the linear velocities coincide, we are able to simplify Eqs. (11) - (13) by reducing them in the order of derivative with the help of the UP approximation [18]. Indeed, suppose that the concentration of the paramagnetic impurities in the crystal is small:

$$
\eta=\frac{n\left(G_{\perp}^{2}+G_{\|}^{2}\right)}{\hbar \omega_{0} \rho a^{2}} \ll 1,
$$

and introduce new independent variables

$$
\tau=t-\frac{z}{a}, \quad \zeta=\eta z
$$

which are usually referred to as the retarded time and slow coordinate, respectively. Obviously, we have

$$
\frac{\partial}{\partial t}=\frac{\partial}{\partial \tau}, \quad \frac{\partial}{\partial z}=-\frac{1}{a} \frac{\partial}{\partial \tau}+\eta \frac{\partial}{\partial \zeta} .
$$

Then, neglecting the terms proportional to $\eta^{2}$, we approximately write

$$
\frac{\partial^{2}}{\partial z^{2}} \approx \frac{1}{a^{2}} \frac{\partial^{2}}{\partial \tau^{2}}-2 \frac{\eta}{a} \frac{\partial^{2}}{\partial \tau \partial \zeta}
$$

in the left-hand side of Eqs. (11)-(13) and

$$
\frac{\partial^{2}}{\partial z^{2}} \approx \frac{1}{a^{2}} \frac{\partial^{2}}{\partial \tau^{2}}
$$

in the right hand side. Integration on $\tau$ of the wave equations obtained by this way in the terms of new variables gives after returning to the initial ones:

$$
\begin{gathered}
\frac{\partial \mathcal{E}_{z z}}{\partial z}+\frac{1}{a} \frac{\partial \mathcal{E}_{z z}}{\partial t}=\frac{n G_{\|}}{4 \sqrt{2} \hbar \rho a^{3}}\left[i\left(\rho_{12}-\rho_{21}+\rho_{23}-\rho_{32}\right)\left(\hbar \omega_{0} \sin 2 \alpha-2 G_{\perp} \mathcal{E}_{x z}\right)+2 G_{\perp}\left(\sqrt{2} \sin \alpha\left(\rho_{33}-\rho_{11}\right)\right.\right. \\
\left.\left.-\cos \alpha\left(\rho_{12}+\rho_{21}+\rho_{23}+\rho_{32}\right)\right) \mathcal{E}_{y z}\right] \\
\begin{array}{r}
\frac{\partial \mathcal{E}_{x z}}{\partial z}+\frac{1}{a} \frac{\partial \mathcal{E}_{x z}}{\partial t}=\frac{n G_{\perp}}{4 \sqrt{2} \hbar \rho a^{3}}\left[i\left(\rho_{12}-\rho_{21}+\rho_{23}-\rho_{32}\right)\left(\hbar \omega_{0} \cos 2 \alpha+G_{||} \mathcal{E}_{z z}\right)+G_{\perp}\left(\sqrt{2} \cos \alpha\left(\rho_{33}-\rho_{11}\right)\right.\right. \\
\left.\left.\left.+\rho_{12}+\rho_{21}+\rho_{23}+\rho_{32}\right)\right) \mathcal{E}_{y z}\right]
\end{array} \\
\begin{array}{r}
\frac{\partial \mathcal{E}_{y z}}{\partial z}+\frac{1}{a} \frac{\partial \mathcal{E}_{y z}}{\partial t}=\frac{n G_{\perp}}{4 \sqrt{2} \hbar \rho a^{3}}\left[\sqrt{2}\left(\rho_{11}-\rho_{33}\right)\left(G_{\perp} \cos \alpha \mathcal{E}_{x z}+G_{\|} \sin \alpha \mathcal{E}_{z z}\right)+\left(\rho_{12}+\rho_{21}+\rho_{23}+\rho_{32}\right)\right. \\
\left.\times\left(\hbar \omega_{0} \cos \alpha-G_{\perp} \sin \alpha \mathcal{E}_{x z}+G_{\|} \cos \alpha \mathcal{E}_{z z}\right)\right]
\end{array}
\end{gathered}
$$


It is assumed hereinafter that the components of the strain field and the density matrix elements satisfy following conditions at $t \rightarrow-\infty$ :

$\mathcal{E}_{x z, y z, z z} \rightarrow 0, \quad \frac{\partial \mathcal{E}_{x z, y z, z z}}{\partial t} \rightarrow 0, \quad \rho_{j j} \rightarrow W_{j}, \quad \rho_{j k} \rightarrow 0$

$(j, k=1,2,3 ; k \neq j)$. These conditions correspond to the pulsed mode of propagation of the sound in a crystal. Note that the evolution equation (7) on density matrix is used to exclude the time derivatives of its elements in the right-hand side of Eqs. (14)-(16).

The equations we came to are yet complicated for the rigorous analysis. Fortunately, they can be reduced in particular case to a system describing the interaction of the transverse-longitudinal acoustic pulses with two-level quantum particles. The investigation of this system will be carried out in the next sections.

\section{SYSTEM OF THE LSWC EQUATIONS}

Let the populations of the quantum states with $S_{z^{\prime}}=$ \pm 1 be equal:

$$
\rho_{33}=\rho_{11} \text {. }
$$

This assumption is compatible with Eqs. (77), (14)- (16) if following conditions are imposed

$$
\rho_{31}=\rho_{13}, \quad \rho_{32}=-\rho_{12}, \quad \mathcal{E}_{y z}=0 .
$$

It is checked immediately that conditions (18) are sufficient to keep (17). Also, they give us relation

$$
\rho_{13}=W_{1}-\rho_{11} \text {. }
$$

Taking into account Eqs. (17)-(19), we rewrite (7) and (14)-116) in the next form:

$$
\begin{gathered}
\frac{\partial W}{\partial t}=i E_{\perp}\left(\sigma-\sigma^{*}\right), \\
\frac{\partial \sigma}{\partial t}=i\left(\omega_{0}+2 E_{||}\right) \sigma+2 i E_{\perp} W \\
\frac{\partial E_{\perp}}{\partial z}+\frac{1}{a} \frac{\partial E_{\perp}}{\partial t}=-i \frac{n A}{4 \hbar \rho a^{3}}\left(\omega_{0}+2 E_{\|}+\delta E_{\perp}\right) \\
\times\left(\sigma-\sigma^{*}\right), \\
\frac{\partial E_{||}}{\partial z}+\frac{1}{a} \frac{\partial E_{||}}{\partial t}=i \frac{n A}{8 \hbar \rho a^{3}}\left[\delta\left(\omega_{0}+2 E_{\|}+\delta E_{\perp}\right)\right. \\
\left.+d^{2} E_{\perp}\right]\left(\sigma-\sigma^{*}\right) .
\end{gathered}
$$

Here

$$
\begin{gathered}
W=\frac{1+W_{2}}{4}-\rho_{22}, \quad \sigma=\sqrt{2} \rho_{21}, \\
E_{\perp}=\frac{G_{\perp}}{\hbar} \cos 2 \alpha \mathcal{E}_{x z}+\frac{G_{\|}}{2 \hbar} \sin 2 \alpha \mathcal{E}_{z z}, \\
E_{\|}=\frac{G_{\|}}{2 \hbar} \cos 2 \alpha \mathcal{E}_{z z}-\frac{G_{\perp}}{\hbar} \sin 2 \alpha \mathcal{E}_{x z}
\end{gathered}
$$

and

$$
\begin{gathered}
A=G_{\perp}^{2} \cos ^{2} 2 \alpha+\frac{G_{\|}^{2}}{2} \sin ^{2} 2 \alpha, \\
\delta=\frac{2 G_{\perp}^{2}-G_{\|}^{2}}{2 A} \sin 4 \alpha, \quad d=\frac{\sqrt{2}}{A} G_{\perp} G_{\|} .
\end{gathered}
$$

System (20)-23) is remarkable from the point of view of its physical applications. In the case $d=\delta=0$ and $E_{\|}=0$, it coincides with well-known reduced MaxwellBloch equations (RMB) for isotropic two-level medium [18]. If $d=0$, then we can put $E_{\|}=-\delta E_{\perp} / 2$, and the system appearing is nothing but the RMB equations for anisotropic medium [19], which describe also the propagation of one-component acoustic pulses through the deformed paramagnetic crystals [1]. At last, Eqs. (20)(23) are equivalent to the system of material and wave equations derived in Ref. 8 under consideration of the dynamics of transverse-longitudinal acoustic pulses in paramagnetic with effective spin $S=1 / 2$ in the external magnetic field presence. The complete investigation of this system turned out to be awkward. The simplest stationary solution found corresponds to the case $\delta=0$ only. It describes the propagation of the extremely short acoustic pulse that possesses no well-defined carrier frequency in direction parallel to magnetic field. In contrast to this, the pulses containing of the higher-frequency components will be considered below.

Owing to the last remark, further simplification of system (20) - 23) will be achieved by applying the SVE approximation. Comparing the right-hand side of Eqs. (22) and (23), we conclude that field $E_{\|}$should have, in the general case, the high-frequency component proportional to $E_{\perp}$. Taking this into account, we make use of the next representation

$$
\begin{gathered}
\sigma=R \exp [i \omega(t-z / a)] \\
E_{\perp}=\left(\Omega_{\perp} / 2\right) \exp [i \omega(t-z / a)]+\text { c.c. }, \\
E_{\|}=\left(\Omega_{\|}-\delta E_{\perp}\right) / 2
\end{gathered}
$$

where $\omega=\omega_{0}-\Delta$ is the carrier frequency $\left(|\Delta| \ll \omega_{0}\right) ; R$, $\Omega_{\perp}$ and $\Omega_{\|}$are slowly varying functions of $t$ and $z$ in the standard sense [20]. Substituting these expressions into Eqs. 201 23) and disregarding the nonresonant terms give us following equations

$$
\begin{gathered}
\frac{\partial W}{\partial t}=\frac{i}{2}\left(\Omega_{\perp}^{*} R-\Omega_{\perp} R^{*}\right), \\
\frac{\partial R}{\partial t}=i\left(\Delta+\Omega_{\|}\right) R+i \Omega_{\perp} W, \\
\frac{\partial \Omega_{\perp}}{\partial z}+\frac{1}{a} \frac{\partial \Omega_{\perp}}{\partial t}=-i \beta_{\perp} R, \\
\frac{\partial \Omega_{\|}}{\partial z}+\frac{1}{a} \frac{\partial \Omega_{\|}}{\partial t}=\beta_{\|} \frac{\partial W}{\partial t},
\end{gathered}
$$


where

$$
\beta_{\perp}=\frac{n \omega_{0} A}{2 \hbar \rho a^{3}}, \quad \beta_{\|}=\frac{n A d^{2}}{4 \hbar \rho a^{3}} .
$$

Note that we neglect the high-frequency addition to detuning $\Delta$ in the right-hand side of Eq. (27). An influence of this term on the dynamics of the pulses, whose duration is much greater than the oscillation period, is not significant because its average value over the pulse length tends to zero. In the case of the RMB equations for anisotropic media [19], this fact was established in Ref. 21.

System (26) - 29) we finally obtain differs only by the notations from the LSWC equations that have been investigated in details due to their importance for theoretical study of the nonlinear dynamics of two-component electromagnetic pulses in the anisotropic resonant media 12, 13. This reveals to us one more correspondence between coherent optical and acoustic phenomena, which will be used in the next section for classification of the modes of the acoustic pulse propagation through strained paramagnetic crystal. It should be noted here that the system of LSWC equations, as well as its gauge equivalents, arises in various physical problems (see, e.g., references in Ref. 13). These numerous applications point to the universal physical character of the LSWC system.

One of the features of Eqs. (26)-29) is that the field variables play in them totally different roles. Namely, $\Omega_{\perp}$ causes the quantum transitions, whereas $\Omega_{\perp}$ shifts dynamically the transition frequency. It is easy to see that there exists relation between the field components:

$$
\Omega_{\|}=-\frac{d^{2}}{4 \omega_{0}}\left|\Omega_{\perp}\right|^{2}+F(t-z / a) .
$$

The last term on the right-hand side generates the phase modulation of $R$ and $\Omega_{\perp}$ only and vanishes after appropriate change of the variables. Without loss of a generality, we put $F(t-z / a)=0$ in what follows.

At the end of this section, we find the expressions for transverse and longitudinal components of the strain field through the variables of the LSWC equations. It follows from Eqs. (24) and 25) that

$$
\begin{array}{r}
\mathcal{E}_{x z}=\frac{\hbar G_{\perp}}{A} \cos 2 \alpha\left(\Omega_{\perp} \exp [i \omega(t-z / a)]+\text { c.c. }\right) \\
-\frac{\hbar}{2 G_{\perp}} \sin 2 \alpha \Omega_{\|}, \\
\mathcal{E}_{z z}=\frac{\hbar G_{\|}}{A} \sin 2 \alpha\left(\Omega_{\perp} \exp [i \omega(t-z / a)]+\text { c.c. }\right) \\
+\frac{\hbar}{G_{\|}} \cos 2 \alpha \Omega_{\|} .
\end{array}
$$

These formulas display that both the components of the acoustic pulse have high-frequency and zero-frequency components in the general case. Also, Eqs. (30)-32) reveal us an asymmetry on the polarity of acoustic signal: the signs of the zero harmonics of its transverse and longitudinal components are determined by $\alpha$ and the type of the external action (tension or compression) on the crystal. It is remarkable that the ratio of the amplitudes of the zero harmonic of $\mathcal{E}_{z z}$ and $\mathcal{E}_{x z}$ :

$$
\frac{G_{\perp}}{G_{\|}} \cot 2 \alpha,
$$

is equal to inverse ratio of the first harmonic case. This fact can be used in measuring the constants of the spinphonon interaction in the paramagnetic crystals.

\section{THE MODES OF ACOUSTIC TRANSPARENCY}

The solution of Eqs. (26) 291 that describes the propagation of the transverse-longitudinal acoustic pulse is written as given (see Ref. 13):

$$
\begin{gathered}
\Omega_{\perp}=\sqrt{M} \exp (i \Phi), \\
\Omega_{\|}=-\frac{d^{2}}{4 \omega_{0}} M, \\
W=\left(1-\frac{\tau_{p}^{2}}{2\left(1+\alpha^{2}\right)} M\right) W_{0} .
\end{gathered}
$$

Here

$$
\begin{gathered}
M=\frac{8 g}{\tau_{p}^{2}\left(g-\alpha+\sqrt{1+(g-\alpha)^{2}} \cosh 2 \zeta\right)}, \\
\Phi=\frac{\beta_{\perp} \alpha \tau_{p}}{1+\alpha^{2}} W_{0} z-\arctan \frac{\tanh \zeta}{s}+\text { const } \\
\alpha=\Delta \tau_{p}, \quad W_{0}=\frac{1-3 W_{2}}{4} \\
g=\frac{2 \omega_{0} \tau_{p}}{d^{2}}, \quad \zeta=\tau_{p}^{-1}\left(t-\frac{z}{v_{g}}\right) \\
s=g-\alpha+\sqrt{1+(g-\alpha)^{2}} \\
v_{g}=a\left(1-\frac{a \beta_{\perp} \tau_{p}^{2}}{1+\alpha^{2}} W_{0}\right)^{-1}
\end{gathered}
$$

The free parameters of the pulse presented are $\Delta$ and $\tau_{p}$. For the sake of convenience we suppose hereinafter that $\tau_{p}>0$. 
As it follows from Eqs. (33) and (37), component $\Omega_{\perp}$ has the phase modulation, which leads to a local nonlinear chirping of the carrier frequency, $\omega \rightarrow \omega_{l o c}=$ $\omega+\delta \omega_{\text {non }}$, where

$$
\delta \omega_{n o n} \equiv \frac{\partial \Phi}{\partial t}=\frac{\Omega_{\|}}{4} .
$$

Taking into account the dynamic shift of the transition frequency $\omega_{0} \rightarrow \omega_{0}^{e f}=\omega_{0}+\Omega_{\|}$(see Eq. (27)), we come to the following expression for effective detuning $\Delta_{e f}$ of component $\Omega_{\perp}$ from the resonance:

$$
\Delta_{e f} \equiv \omega_{0}^{e f}-\omega_{l o c}=\Delta+\frac{3}{4} \Omega_{\|} .
$$

Defining the pulse length $T_{p}$ as the double deviation from the zero point of $t-z / v_{g}$, at which $\left|\Omega_{\perp}\right|$ is half its maximum value, we obtain from formula (333):

$$
T_{p}=\tau_{p} \operatorname{arccosh}\left(4+3 \frac{g-\alpha}{\sqrt{1+(g-\alpha)^{2}}}\right) .
$$

In the SVE approximation, both pulse length and nonlinear shift of the carrier frequency must obviously satisfy the conditions $\omega_{0} T_{p} \gg 1$ and $\left|\delta \omega_{n o n}\right| \ll \omega_{0}$. It can easily be shown that these inequalities are valid if $\omega_{0} \tau_{p} \gg 1$ and $\omega_{0} \tau_{p} \gg \alpha-g$. The last condition is necessary in the case $\alpha-g \gg 1$, evidently, and can be fulfilled only when $d^{2} \gg 1$.

Considering the limit $\tau_{p} \rightarrow \infty$ in formulas (33) -38) gives us rationally decreasing pulse solution:

$$
\begin{aligned}
& \Omega_{\perp}=\frac{8 i \omega_{0} \kappa}{d^{2}\left(1+i \kappa^{2} \xi\right)} \exp \left(i \beta_{\perp} W_{0} z / \Delta\right), \\
& W=\left(1-\frac{8 \kappa^{2}}{\left(1+\kappa^{2}\right)^{2}\left(1+\kappa^{4} \xi^{2}\right)}\right) W_{0},
\end{aligned}
$$

where

$$
\begin{gathered}
\xi=\frac{4 \omega_{0}}{d^{2}}\left(t-\frac{z}{v_{r}}\right), \quad \kappa=\sqrt{\frac{d^{2}}{2 \omega_{0}} \Delta-1,} \\
v_{r}=a\left(1-\frac{a \beta_{\perp}}{\Delta^{2}} W_{0}\right)^{-1} .
\end{gathered}
$$

Parameter $\kappa$ in Eqs. (42) and (43) is supposed to be real. This imposes a constraint on detuning $\Delta$ of the rationally decreasing pulses: $\Delta>\Delta_{r}$, where $\Delta_{r}=2 \omega_{0} / d^{2}$.

Now, we use the expressions presented above to identify the modes of the propagation of transverselongitudinal acoustic pulses in deformed paramagnetic crystal. These modes resemble the ones studied in Ref. 13 for the case of two-component electromagnetic pulses. In what follows, it is assumed for the sake of concreteness that the paramagnetic impurities are in thermodynamic equilibrium prior to the pulse passage $\left(-1 / 2<W_{0}<0\right)$.
Let us begin with the modes, in which the pulse excites the paramagnetic impurities strongly. Since $W^{2}+|R|^{2}$ is independent of $t$, the strongest degree of excitation happens in the case, when the value of variable $W$ in the pulse center differs by the sign from its value at absence of the pulse. By virtue of Eqs. (35) and (36), this condition can be formulated as

$$
\tau_{p}^{2}\left|\Omega_{\perp}(\zeta=0)\right|^{2}=4\left(1+\alpha^{2}\right)
$$

Performing some simple algebra, we put it into the next form

$$
\left(\alpha^{2}-2 g \alpha+1\right)^{2}=0
$$

which yields

$$
g=\frac{1}{2}\left(\alpha+\frac{1}{\alpha}\right)
$$

This condition can take place if $\alpha>0$ only. It can also be rewritten as a relation between the pulse detuning $\Delta$ and parameter $\tau_{p}$ :

$$
\tau_{p}^{-1}=\sqrt{\frac{4 \omega_{0}}{d^{2}} \Delta-\Delta^{2}}
$$

Setting the right-hand side to zero, we find the interval of admissible values of the detuning: $0<\Delta<\Delta_{m}=$ $2 \Delta_{r}$. The curve of the strong excitation (45) is plotted in Fig. 3.

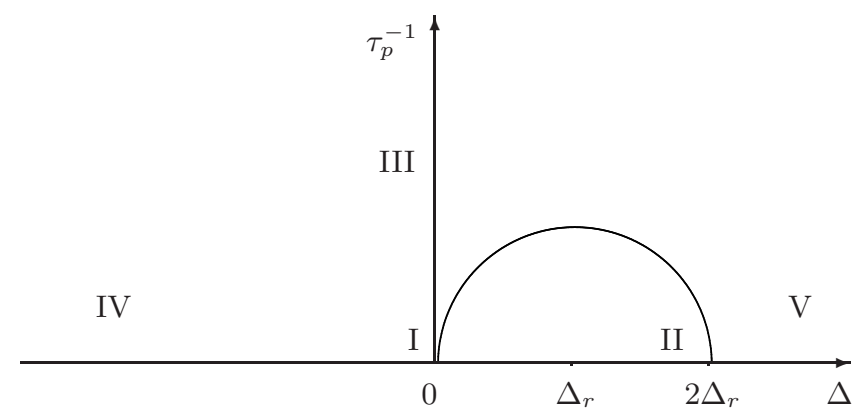

FIG. 3: Curve of the strong excitation. Domains of the pulse parameters corresponding to different modes of acoustic transparency: (I) ASIT; (II) ASIST; (III) AEOT; (IV) ANNT; (V) APNT.

It is seen from Eq. (43) that the strongest excitation in the case of rationally decreasing pulse takes place if $\kappa=1$. Obviously, this agrees with (45). 
Substitution of relation (44) into Eqs. (33)-(38) yields

$$
\begin{gathered}
\left|\Omega_{\perp}\right|=\frac{2 \sqrt{1+\alpha^{2}}}{\tau_{p} \sqrt{1+\left(1+\alpha^{2}\right) \sinh ^{2} \zeta}}, \\
\Omega_{\|}=-\frac{4 \Delta}{1+\left(1+\alpha^{2}\right) \sinh ^{2} \zeta}, \\
W=\left(1-\frac{2}{1+\left(1+\alpha^{2}\right) \sinh ^{2} \zeta}\right) W_{0}, \\
v_{g}=a\left(1-\frac{a \beta_{\perp} \tau_{p}^{2}}{1+\alpha^{2}} W_{0}\right)^{-1} .
\end{gathered}
$$

The corresponding pulse length is

$$
T_{p}=2 \tau_{p} \operatorname{arcsinh} \sqrt{\frac{3}{1+\alpha^{2}}} .
$$

Accordingly to Eqs. (40) and (46), the effective detuning of $\Omega_{\perp}$ from the resonance equals $\Delta$ at the edges of the pulse and $-2 \Delta$ at its center. Thus, we can say that this component is resonant with quantum transitions on average over the pulse length. As a result, the largest possible change in the population of the spin sublevels is achieved.

If the detuning is very small $(\alpha \ll 1, g \gg 1)$, then Eqs. (46) can be represented as

$$
\begin{gathered}
\left|\Omega_{\perp}\right|=\frac{2}{\tau_{p}} \operatorname{sech} \zeta, \quad \Omega_{\|}=-4 \Delta \operatorname{sech}^{2} \zeta, \\
W=\left(1-2 \operatorname{sech}^{2} \zeta\right) W_{0}, \\
v_{g}=a\left(1-a \beta_{\perp} \tau_{p}^{2} W_{0}\right)^{-1} .
\end{gathered}
$$

One can see that $\left|\Omega_{\|}\right| \ll\left|\Omega_{\perp}\right|$, and the phase-modulation depth for component $\Omega_{\perp}$ is much smaller than its input spectral width: $\left|\delta \omega_{\text {non }}\right| \ll 1 / \tau_{p}$. In the exact resonance case $(\alpha=0, g \rightarrow \infty)$, these relations give us well-known expressions of the ASIT theory 3, 4, 5].

Formulas (46) and (39) show that an increase of detuning (and therefore $\alpha$ ) leads to larger amplitudes of the pulse components and to a deeper chirping of $\Omega_{\perp}$ toward lower frequencies. Since the pulse length decreases, the profiles of both components become sharper.

Also, it is necessary to note that the group velocity approaches linear velocity $a$ of acoustic wave as detuning increases. Nevertheless, the strong excitation of the paramagnetic impurities takes place: the largest possible change in the level population is reached at the center of the pulse. This is evidently explained by the fact that a growth in the amplitude of pulse increases its power. The ensuing higher rate of excitation/de-excitation processes leads to a higher soliton propagation velocity.

It is clear from (44) that each particular value of $g$ corresponds to two distinct values of $\alpha$. We associate the domain of relatively small detuning $(\alpha<1)$ with the ASIT mode, since it is implemented in the case $\alpha=0$. When $\alpha>1$, we say that the pulse solutions given by
(46) propagate in the mode of acoustic self-induced supertransparency (ASIST), thus emphasizing the fact that the group velocity decrease is lower as compared to the ASIT mode while excitation is equally strong. Since $\left|\Delta_{e f}\right| \ll \omega_{0}$ in the SVE approximation, the ASIST mode must be most strongly manifested if $d^{2} \gg 1$. The domains of the values of the pulse parameters, which correspond to these modes, are schematized in Fig. 3.

When $g \gg 1$ and $\alpha \gg 1$, the amplitudes of components $\Omega_{\perp}$ and $\Omega_{\|}$of the ASIST pulse and the phase-modulation depth for $\Omega_{\perp}$ reach their limits $2 \Delta_{m}, 4 \Delta_{m}$ and $\Delta_{m}$, respectively. In this case, (47) gives us the following estimate for the pulse length:

$$
T_{p} \approx \frac{2 \sqrt{3} \tau_{p}}{\alpha} \approx \frac{d^{2}}{\omega_{0}} .
$$

This time scale corresponds to the time scale of phasemodulation localization. A deeper phase modulation combined with a decrease in the corresponding localization time scale and a shorter pulse length can be interpreted as an effect of the spectral supercontinuum generation. Indeed, the Fourier transform of (33) and (34) defined as

$$
F_{\perp,||}(\nu)=\int_{-\infty}^{\infty} \mathrm{e}^{i \nu t} \Omega_{\perp, \|} d t
$$

yields

$$
\begin{gathered}
\left|F_{\perp}(\nu)\right|=2 \pi \frac{\sqrt{g}}{\sqrt[4]{1+(g-\alpha)^{2}}} \frac{\mathrm{e}^{\theta \tau_{p} \nu / 2}}{\cosh \left(\pi \tau_{p} \nu / 2\right)}, \\
\left|F_{||}(\nu)\right|=4 \pi \frac{\sinh \left(\theta \tau_{p} \nu / 2\right)}{\sinh \left(\pi \tau_{p} \nu / 2\right)}
\end{gathered}
$$

where $\theta=\operatorname{arccot}(g-\alpha)(0<\theta<\pi)$. The absolute values of the Fourier transforms of the components $\Omega_{\|}$ and $\Omega_{\perp}$ reach their maximum values at $\nu=0$ and $\nu=\nu_{0}$, respectively, where

$$
\nu_{0}=\frac{2}{\pi \tau_{p}} \ln \frac{\pi+\theta}{\pi-\theta} .
$$

If $\alpha-g \gg 1$ or, equivalently, $\theta \rightarrow \pi$, then the spectral width of the pulse is $\delta \omega \sim 1 /(\pi-\theta) \tau_{p}$ and the maximum of the spectral energy distribution of component $\Omega_{\perp}$ is reached at frequency $\omega-\nu_{0}$, which is much lower than the carrier frequency $\omega$. In this case, despite a large linear detuning from resonance $\left(\tau_{p} \Delta \gg 1\right)$, a substantial nonlinear spectral broadening $\left(\tau_{p} \delta \omega \gg 1\right)$ and dynamical shift in the transition frequency $\omega_{0} \rightarrow \omega_{0}^{\text {ef }}$ lead to a generation of the resonant Fourier components (photons), which stimulate the quantum transitions.

Let us now consider the modes of the acoustic pulse propagation in the case $g \ll 1$. Although the paramagnetic impurities are excited weakly under this condition, 
there exist remarkable features in their interaction with the acoustic pulses. Since $\omega_{0} \tau_{p} \gg 1$ in the SVE approximation, the value of $|d|$ must be sufficiently large to ensure that $\omega_{0} \tau_{p} / d^{2} \ll 1$. This can be fulfilled for some values of $\alpha$ if $\left|G_{\perp}\right| \gg\left|G_{\|}\right|$or vice versa.

First, we assume that the detuning from resonance is large $(|\alpha| \gg 1)$. If $\alpha<0$ (or $\left.\omega>\omega_{0}\right)$, then Eqs. (33)(38) give

$$
\begin{gathered}
\left|\Omega_{\perp}\right|=\frac{2}{\tau_{p}} \sqrt{\frac{g}{|\alpha|}} \operatorname{sech} \zeta, \\
\Omega_{\|}=-\frac{2}{|\alpha| \tau_{p}} \operatorname{sech}^{2} \zeta, \\
W=\left(1-\frac{2 g}{|\alpha|^{3}} \operatorname{sech}^{2} \zeta\right) W_{0}, \\
v_{g}=v_{r} .
\end{gathered}
$$

Comparing these expressions with (48), we see that the amplitude of $\Omega_{\perp}$ is much smaller than in the ASIT mode, whereas the respective $\Omega_{\| \mid}$'s are comparable. We also note that

$$
\frac{\left|\Omega_{\perp}(\zeta=0)\right|}{\left|\Omega_{||}(\zeta=0)\right|}=\sqrt{g|\alpha|}
$$

i.e., the amplitude ratio can have an arbitrary value. The paramagnetic impurities remain almost unexcited as the soliton described by (49) propagates through the crystal, and the soliton velocity decreases only very slightly. The phase-modulation depth is also small $\left(\left|\delta \omega_{n o n}\right| \ll 1 / \tau_{p}\right)$, and the effective detuning $\Delta_{e f}$ only increases as the component $\Omega_{\|}$is generated. This leads to an even weaker excitation of the impurities as compared to that induced by the input pulse.

Now, let us suppose that $g \ll 1$ and $\alpha \gg 1$. In this case Eqs. (33)-38) imply

$$
\begin{gathered}
\left|\Omega_{\perp}\right|=\frac{4 \sqrt{g \alpha}}{\tau_{p} \sqrt{1+4 \alpha^{2} \sinh ^{2} \zeta}}, \\
\Omega_{\|}=-\frac{8 \Delta}{1+4 \alpha^{2} \sinh ^{2} \zeta}, \\
W=\left(1-\frac{8 g}{\alpha\left(1+4 \alpha^{2} \sinh ^{2} \zeta\right)}\right) W_{0}, \\
v_{g}=v_{r} .
\end{gathered}
$$

Note that expressions (46) and (50) are somewhat similar: in both cases, the solitons are sharply peaked, and their propagation velocities are nearly equal to $a$. However, these modes are essentially different in terms of behavior of the paramagnetic impurities. Whereas they are strongly excited as the soliton described by (46) propagates through crystal, no significant excitation is caused in the case of (50). Indeed, since the effective detuning $\Delta_{e f}$ of the pulse described by Eqs. (50) is $-5 \Delta$ at its center, it is not resonant with the paramagnetic impurities on average over the pulse length. However, according to (49) and (50), the excitation of the paramagnetic impurities at $\omega<\omega_{0}$ being relatively weak, is still stronger than that at $\omega>\omega_{0}$. The reason is that the effective detuning decreases toward the pulse center when $\omega<\omega_{0}$, owing to the component $\Omega_{\|}$, and increases when $\omega>\omega_{0}$. Thus, a comparison of (49) with (50) demonstrates obvious asymmetry with respect to detuning of $\Omega_{\perp}$. Since $\alpha<0$ for the pulses described by (49) and $\alpha>0$ for ones described by (50), we refer to the corresponding modes as acoustic negative and positive nonresonant transparency (ANNT and APNT), respectively.

If $g \ll 1$ and $|\alpha| \ll 1$ (detuning is small), then Eqs. (33) - (38) lead to expressions identical to those found in Ref. 12:

$$
\begin{gathered}
\left|\Omega_{\perp}\right|=\frac{2}{\tau_{p}} \sqrt{2 g} \operatorname{sech}^{1 / 2} 2 \zeta, \\
\Omega_{\|}=-\frac{4}{\tau_{p}} \operatorname{sech} 2 \zeta, \\
W=(1-4 g \operatorname{sech} 2 \zeta) W_{0} .
\end{gathered}
$$

The expression for group velocity coincides with one given in Eqs. (48) corresponding to the ASIT mode.

Here, we have $\left|\Omega_{||} / \Omega_{\perp}\right|^{2} \gg 1$ in the center of the pulse. The paramagnetic impurities are not excited, since the effective detuning is large, $3 / \tau_{p}$. However, the propagation velocity decreases as in the case of strong excitation at $\alpha \sim 1$. Such a deceleration of the pulse is explained by the dispersion properties of medium within the higher-frequency component bandwidth. According to [12], where this effect was studied in details for the optical solitons, we call the mode considered an acoustic extraordinary transparency (AEOT). The roles of the ordinary and extraordinary pulse components are played here by $\Omega_{\perp}$ and $\Omega_{\|}$, respectively. The existence of this mode for transverse-longitudinal acoustic pulses has been revealed also in the case, when the splitting of the spin sublevels is produced by the external magnetic field [7, 10]. The domains of existence of the modes with $g \ll 1$ are presented in Fig. 3.

It should be noted that expressions (33)-38) do not change their form in a presence of detuning between $a_{\|}$ and $a_{\perp}$. The influence of this detuning is inessential if condition

$$
\varepsilon \equiv\left(\frac{1}{a_{\perp}}-\frac{1}{a_{\|}}\right)\left(\frac{1}{v_{g}}-\frac{1}{a}\right)^{-1} \ll 1
$$

is valid. Substituting the definitions for $\beta_{\perp}$ and $A$ into (38), we obtain

$$
\varepsilon \sim \frac{1}{a \beta_{\perp} \tau_{p}^{2}}\left(\frac{1}{a_{\perp}}-\frac{1}{a_{\|}}\right) \sim \frac{\hbar \rho a_{\|}^{2}}{n G_{\|}^{2} \omega_{0} \tau_{p}^{2}}\left(\frac{a_{\|}}{a_{\perp}}-1\right) .
$$

Taking for $\mathrm{Fe}^{2+}: \mathrm{MgO}$ 3, 6, 14, 22, 23] $n \sim 10^{17} \mathrm{~cm}^{-3}$, $\omega_{0} \sim 10^{10} \mathrm{~s}^{-1}, G_{\|} \sim 10^{-13} \mathrm{erg}, \rho \approx 2 \mathrm{~g} / \mathrm{cm}^{3}, a_{\|} \sim$ $5 \cdot 10^{5} \mathrm{~cm} / \mathrm{s}, a_{\|} / a_{\perp} \sim 1.5$ and $\tau_{p} \sim 10^{-8}$ s, we find $\varepsilon \sim 0.1$. This estimation shows that we can neglect detuning between the linear velocities of transverse and longitudinal acoustic waves for typical values of the ASIT parameters. 
TABLE I: Characteristics of the modes of acoustic transparency

\begin{tabular}{l|c|c|c|c|c|l}
\hline Mode & $\tilde{\Delta}_{e f}^{a}$ & $|\alpha|$ & $g$ & $v_{g}$ & $\left|\Omega_{\perp} / \Omega_{\|}\right|$ & $\begin{array}{c}\text { Excita- } \\
\text { tion }\end{array}$ \\
\hline ASIT & $-2 \Delta$ & $\ll 1$ & $\gg 1$ & $v_{A S I T}$ & $\gg 1$ & strong \\
\hline ASIST & $-2 \Delta$ & $\gg 1$ & $\gg 1$ & $<a$ & $\sim 1$ & strong \\
\hline ANNT & $\approx \Delta$ & $\gg 1$ & $\ll 1$ & $a$ & arbitrary & weak \\
\hline APNT & $-5 \Delta$ & $\gg 1$ & $\ll 1$ & $a$ & $\ll 1$ & weak \\
\hline AEOT & $\gg|\Delta|$ & $\ll 1$ & $\ll 1$ & $>v_{A S I T}$ & $\ll 1$ & weak \\
\hline
\end{tabular}

${ }^{a}$ Note: $\tilde{\Delta}_{e f}$ is the effective detuning of the high-frequency components at the center of acoustic pulse; $\tilde{\Delta}_{e f}$ is compared with $\Delta$ in the AEOT mode on absolute value.

\section{CONCLUSION}

In this paper, we have investigated the soliton modes of the acoustic transparency in a strained cubic crystal containing the resonant paramagnetic impurities with effective spin $S=1$. It is supposed that the linear velocities of transverse and longitudinal sound are close, and the pulses propagate through the crystal under arbitrary angle with respect to the direction of external deformation parallel to the fourth-order symmetry axis.

We have allocated five modes of acoustic transparency, which differ by the propagation velocity of the transverselongitudinal pulses and degree of excitation of the paramagnetic impurities (see Table I). The acoustic self-induced transparency is characterized by strong excitation and substantial deceleration in the pulse propagation velocity relative to linear velocities. Self-induced supertransparency differs from ASIT in that the decrease of velocity is small, but the paramagnetic impurities are strongly excited as well. The solitons of ASIST have larger amplitudes and smaller lengths as compared to ASIT solitons, and their high-frequency spectral components are strongly modulated. The carrier frequency of the trans- verse and longitudinal components in this mode is lower than the resonant frequency. The modes, in which the trapping of the populations of the spin sublevels takes place, are also identified. The pulses propagating in the acoustic extraordinary transparency mode are characterized by small detuning of the high-frequency components and dominant role of the zero-frequency ones. Their group velocity substantially changes and may become comparable to that of pulses in ASIT and ASIST modes. In the acoustic positive and negative nonresonant transparency modes, the pulse velocity changes insignificantly, and the absolute value of detuning is large. The most substantial difference between these modes concerns the behavior of the effective detuning of the high-frequency components. In the ANNT mode, it remains virtually constant. If a pulse propagates in the APNT mode, then the effective detuning changes sign due to an influence of the zero-frequency component. Since the local frequency passes through a resonance, a slightly stronger excitation occurs in this case, and the pulses are sharply peaked, as in the ASIST mode.

In this study, we ignored inhomogeneous broadening of the spin sublevels. An investigation allowing for this effect may throw light on the pulse area theorem, providing a basis for analysis of the stability of the transverselongitudinal acoustic pulses. Also, it would be interesting to identify the distinctive features of the dynamics of picosecond transverse-longitudinal acoustic pulses in a system of effective spins $S=1$. Unlike the present consideration, the SVE approximation is inapplicable to this case.

\section{ACKNOWLEDGMENT}

This study was supported by the Russian Foundation for Basic Research (Grant No. 05-02-16422).
[1] S.L. McCall, and E.L. Hahn, Phys. Rev. Lett. 18, 908 (1967).

[2] S.L. McCall, and E.L. Hahn, Phys. Rev. 183, 457 (1969).

[3] N.S. Shiren, Phys. Rev. B 2, 2471 (1970).

[4] G.A. Denisenko, Sov. Phys.-JETP 33, 1220 (1971).

[5] V.V. Samartsev, B.P. Smolyakov, and R.Z. Sharipov, JETP Lett. 20, 296 (1974).

[6] J.W. Tucker, and V.W. Rampton, Microwave Ultrasonics in Solid State Physics (North-Holland, Amsterdam, 1972).

[7] S.V. Voronkov, and S.V. Sazonov, JETP 93, 236 (2001).

[8] A.A. Zabolotskii, JETP 96, 1089 (2003).

[9] A.A. Zabolotskii, Phys. Rev. E 67, 066606 (2003).

[10] A.V. Gulakov, and S.V. Sazonov, J. Phys.: Condens. Matter 16, 1733 (2004).

[11] S.V. Sazonov, and N.V. Ustinov, JETP 102, 741 (2006).

[12] S.V. Sazonov, JETP 97, 722 (2003).

[13] S.V. Sazonov, and N.V. Ustinov, JETP 100, 256 (2005).
[14] S.V. Sazonov, JETP 91, 16 (2000).

[15] E.H. Jacobsen, and K.W.H. Stevens, Phys. Rev. 129, 2036 (1963).

[16] S.V. Sazonov, J. Phys.: Condens. Matter 6, 6295 (1994).

[17] Ch. Kittel, Introduction to Solid State Physics (Wiley, New York, 1956).

[18] J.C. Eilbeck, J.D. Gibbon, P.J. Caudrey, and R.K. Bullough, J. Phys. A 6, 1337 (1973).

[19] M. Agrotis, N.M. Ercolani, S.A. Glasgow, and J.V. Moloney, Physica D 138, 134 (2000).

[20] L. Allen, and J.H. Eberly, Optical Resonance and TwoLevel Atoms (Wiley, New York, 1975).

[21] S.V. Sazonov, and N.V. Ustinov, Quantum Electronics 35, 701 (2005).

[22] A.M. Portis, Phys. Rev. 91, 1071 (1953).

[23] S.V. Sazonov, JETP Lett. 76, 143 (2002). 